\title{
STUDY ON THE CRITICAL VELOCITY OF GROUNDWATER TO FORM SUBSIDENCE SINKHOLES IN A KARST AREA
}

\author{
Fuwei Jiang \\ School of Resources and Environmental Engineering, Guizhou Institute of Technology, No.1, Caiguan Road, Yunyan \\ District, Guiyang City, Guizhou Province, China; Institute of Karst Geology, CAGS, No.50, Qixing Road, GuilCity, \\ Guangxi Province, China, jfwei_666@126.com
}

Mingtang Lei

Institute of Karst Geology, CAGS, No.50, Qixing Road, GuilCity, Guangxi Province, China, mingtanglei@hotmail.com

Jian-ling Dai

Institute of Karst Geology, CAGS, No.50, Qixing Road, GuilCity, Guangxi Province, China, 147538422@qq.com

\begin{abstract}
Subsidence sinkholes in a karst area are a common geological hazard causing disaster accidents. However, the critical hydraulic conditions of forming subsidence sinkholes have not been well understood. Based on the theories of pipe flow, this paper derives expressions of a critical hydraulic condition for assessing whether it leads to subsidence sinkholes. A case study with samples of the cohesive soils taken from the Wuxuan county, Guangxi province, China, was conducted to evaluate the derived critical value. Combining the derived critical value and the monitoring data of hydraulic conditions in the study area our results indicate that subsidence sinkholes are not forming under the current hydraulic conditions.
\end{abstract}

\section{Introduction}

Subsidence sinkholes in karst terrain are a common geological hazard, which can lead to natural disasters in the natural and built environment. One of the main triggering factors of subsidence sinkholes is groundwater. The groundwater alterations may trigger or accelerate sinkhole formation. But it is difficult to obtain the critical condition of the groundwater variation to form subsidence sinkholes. The objective of this study is to discuss the issue.

\section{Hydrodynamic condition}

Subsidence sinkholes are mainly caused by water seepage. In a seepage process, water seeps into soil through the pores (Figure 1a), and it takes the shear stress on the soil grains of the pore wall. When the shear stress is enough to break the soil, it leads to soil-caves. It is hard to make the properties of water seeping into the soil pores clear, as the natural pores of soil are irregular. Ideally, the study makes the soil pores to be regular circular pipes with certain diameters (Figure 1a).
Therefore, water seepage into soil pores is equivalent to water flow in pipes (Figure 1b), which is in accordance with the piping flow theories.

Assuming that water seeps into pores its characteristics can be analyzed with the piping flow theories in order to obtain the critical values of suffosion. An arbitrary micro-unit of the soil cross section is selected as the study object, and an irregular pore of soils is treated as the pipe of $d$ in diameter showen in Figure 1b. Ac-
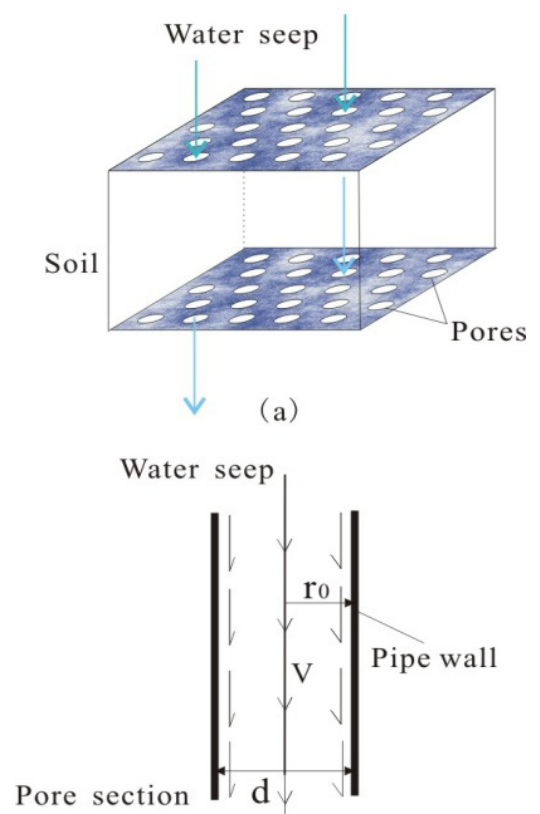

(b)

Figure 1. The process of water seepage into soil: $\mathbf{a}$. water seep into soil layer; $\boldsymbol{b}$. water seep through pores. 
cording to the piping flow theories, the water shear stress to the pore wall can be formulated as:

$$
\tau=\chi R I
$$

Where $\gamma$ is water unit weight; $\mathrm{R}$ is hydraulic radius; $\mathrm{I}$ is hydraulic gradient in the pipe. According to the Newton Inner Friction Law, it can be formulated as follows:

$$
\tau=-\mu \frac{d V_{z}}{d r}
$$

Where $\mu$ is the dynamic viscosity coefficient of water, and $\mathrm{Vr}$ is the flow velocity at $\mathrm{r}$ away from the pipe axis. According to the boundary conditions, the pipe wall velocity is 0 :

$$
\left.V_{\mathrm{r}}\right|_{\mathrm{r}=\mathrm{I}_{0}}=0
$$

After integration, it can be defined as:

$$
V_{\mathrm{r}}=\frac{\gamma}{4 \mu}\left(\mathrm{r}_{0}^{2}-\mathrm{r}^{2}\right)
$$

The mean velocity is:

$$
\bar{V}=\frac{Q}{A}=\frac{\int_{0}^{2} 2 \pi \mathrm{rvdr}}{\pi \mathrm{r}_{0}^{2}}=\frac{\gamma}{8 \mu} \mathrm{r}_{0}^{2}
$$

It can be further calculated as:

$$
I=\frac{8 \mu \bar{V}}{\gamma r_{0}^{2}}
$$

For the pipe:

$$
R=\frac{\mathrm{r}_{0}}{2}=\frac{\mathrm{d}}{4}
$$

After the substitution of formula 7 :

$$
I=\frac{32 \mu \bar{V}}{\gamma \mathrm{d}^{2}}
$$

Where $d$ is the diameter of the pipe. After the introduction of the critical seepage quantity:

$$
Q_{\mathrm{cr}}=\frac{\pi \mathrm{d}^{2}}{4} \bar{V}_{\mathrm{cr}}
$$

After the combination of formulas (1), (8) and (9), the critical shear stress, mean flow velocity and hydraulic gradient are obtained (Jiang 2014):

$$
\tau_{\mathrm{cr}}=\frac{32 \mu}{\pi} \cdot \frac{Q_{\mathrm{cr}}}{\mathrm{d}^{3}}
$$

$$
\bar{V}_{\mathrm{cr}}=\frac{\tau_{\mathrm{cr}} \mathrm{d}}{8 \mu}
$$

In the seepage process, if the critical shear stress-which can cause suffosion-is $\mathrm{F}$ it is correlated with the acting force between soil grains (or aggregates). $\mathrm{F}$ is an inherent attribute which is uncorrelated with the fluid and the pipe diameter. When the fluid shear stress reaches the clay critical shear stress $\mathrm{F}$ the soil grains separate and move away. Under ideal conditions (the identical properties, the constant acting force between the wall grains, and the uniform distribution) clays have an invariant critical shear stress in the seepage process of pores with different sizes. The following is a formula based on equation 11 , with different pipe diameters, i.e. $d_{1}$ and $d_{2}$ (Jiang 2014):

$$
\tau_{c x}=\frac{32 \mu}{\pi} \cdot \frac{Q_{1 c x}}{\mathrm{~d}_{1}{ }^{3}}=\frac{32 \mu}{\pi} \cdot \frac{Q_{2 c r}}{\mathrm{~d}_{2}{ }^{3}}
$$

Formula 12 indicates that the critical shear stress can be obtained by simulating suffosion in large diameter pipes of soil, and then the critical hydraulic conditions of different small diameter pores can be worked out. Based on this principle, the critical shear stress can be tested by means of the seepage of large diameter pores.

\section{Test methods \\ Test procedure}

The pipe flow test device (Figure 2) can be divided into two main parts: hydrodynamic system and sample pot 


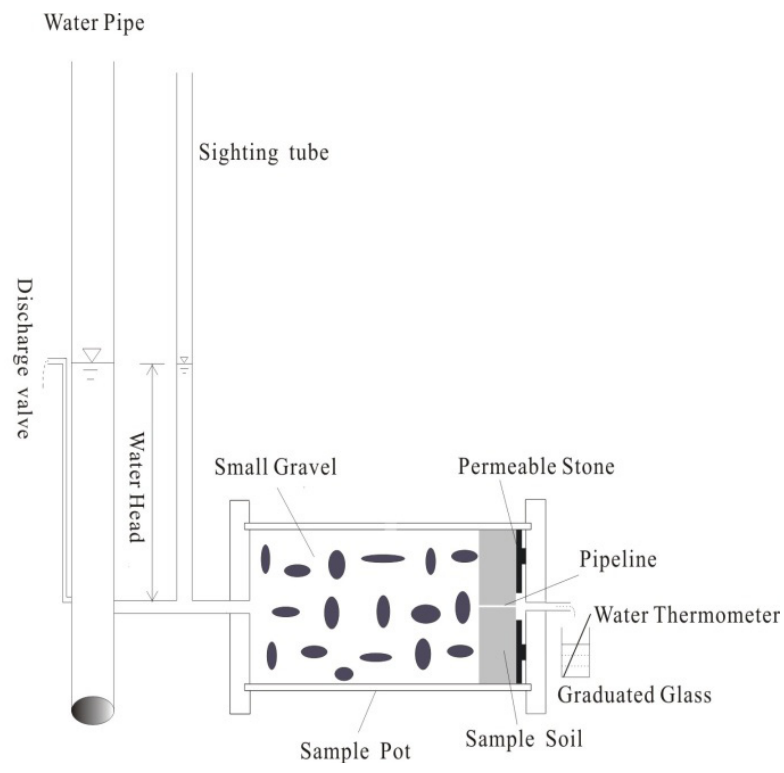

Figure 2. Device of piping flow, which is modified according to the pinhole test.

system, simulating seepage hydraulic pressure and pipe flow respectively. The hydrodynamic system is mainly composed of a water pipe and discharge valve. Water is filled in the water pipe, which raises the water level. The height of the drain valve and the water inflow are adjusted to maintain a constant seepage pressure, which can be read through the sighting tube.

The sample pot is modified in the pinhole test device. Small gravels are put into the pot to avoid the water flow rushing right into the sample. There are permeable stones between the sample and the tail cover, preventing the wall effect at the tail. Pores of a certain diameter are pierced through the middle of the sample to simulate pipes.

The procedure of experiment includes the following six steps:

a) Sample preparation: the sample is $90 \mathrm{~mm}$ in diameter and $38.1 \mathrm{~mm}$ in thickness, and the pipe is $1 \mathrm{~mm}$ in diameter.

b) Follow the schematic diagram to install the device.

c) Saturate the sample.

d) Increasing the water head: fix the drain valve at the level of the starting water head, and fill water into the water pipe until the water runs out from the drain valve. Use the same method to increase the head with $10 \mathrm{~cm}$ increments until soil failure. Keep heads for 30 minute intervals. e) Measurement and observation: It is crucial to measure the flow quality and water temperature accurately. Then observe the seeping phenomenon every 10 minutes, such as the permeating capacity of water and water turbidity, bubbles, and flowing particle, and record to the corresponding water head.

f) Test termination: When the soil breaks through to the phenomenon of suffosion, e.g. particle loss, water head drastically decreases suddenly, water turbidity increases, etc., the test is terminated.

\section{Critical seepage quantity}

Before the sample becomes eroded, the seepage quantity adjusts itself to the ups and downs of the seepage water head value, with roughly the same increase extent. During the variation, slight turbidity and deposition occur in the flow water. Occasionally, the seepage quantity decreases as the seepage water head increases. This is because the debris, which are formed in the process of sample preparation and pipe piercing, are eroded and washed away by the water flow making the pipe clogged up. The distribution of the sample data can be seen in the unbroken state curve in the semilogarithmic plot in Figure 3.

When the sample is eroded, the seepage quantity generally becomes turbid and has coarse sediment grains, the seepage quantity increases significantly, and the seepage water head changes. It can be viewed as suffosion: a) when the seepage quantity increases, with the difference of the increase between seepage quantity and seepage

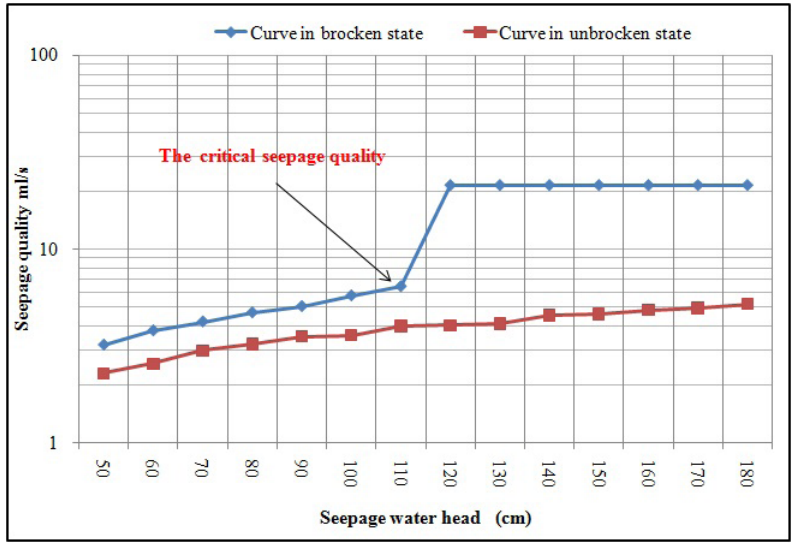

Figure 3. Semilogarithmic plot in the broken or unbroken state. 
head more than $20 \%$ (the seepage quantity increases with the water head, with a typical increase extent of about $10 \%$. Accordingly, an increase extent of more than $20 \%$ can be recommended to be one of the evidences for suffosion.); b) the seepage water head decreased greatly; c) the flow water becomes turbid; d) the coarse sediment grains appear. In the semilogarithmic plot, the seepage discharge manifests itself as stair-like upward inflection points (as shown in the broken state curve in Figure $3)$. After the test, it is found that, the pipe diameter of the pipe above the water becomes larger and grains are washed away (Figure 4a, b).

After the suffosion, the value of the critical seepage quantity can be estimated as that of the previous seepage quantity, namely, the low value of the inflection points shown in Figure 3.

\section{Application in Wuxuan}

Wuxuan is located at the center of Guangxi province, China (N2335'53.57", E109³9'34.43"), which strongly develops karst and is covered by clay. It has the proper geological condition to form sinkholes.

The soil sample was taken undisturbed from a borehole (ZK5), Specimens were extracted at the bottom of layer of a depth 9.0-10.0 m. Three samples were tested to obtain the critical shear stress.

\section{Critical shear stress}

Table 1 shows the test result according the test method mentioned above. The critical seepage quantity is from $1.754 \mathrm{ml} / \mathrm{s}$ to $1.806 \mathrm{ml} / \mathrm{s}$. Water temperature is also tested to obtain the dynamic viscosity coefficient. Re (Reynolds number) shows water flow in the test is in a state of laminar flow $(\operatorname{Re}<2000)$. Therefore, it calculates the critical shear stress effectively by equation 12 . The mean value $21.5 \mathrm{~Pa}$ is the critical shear stress of clay in the study area.

\section{Pores diameter}

Pore diameters were measured by a scanning electron microscope. The circle equivalent diameters of 43 pores were measured in vision field of 120,000 times mag-
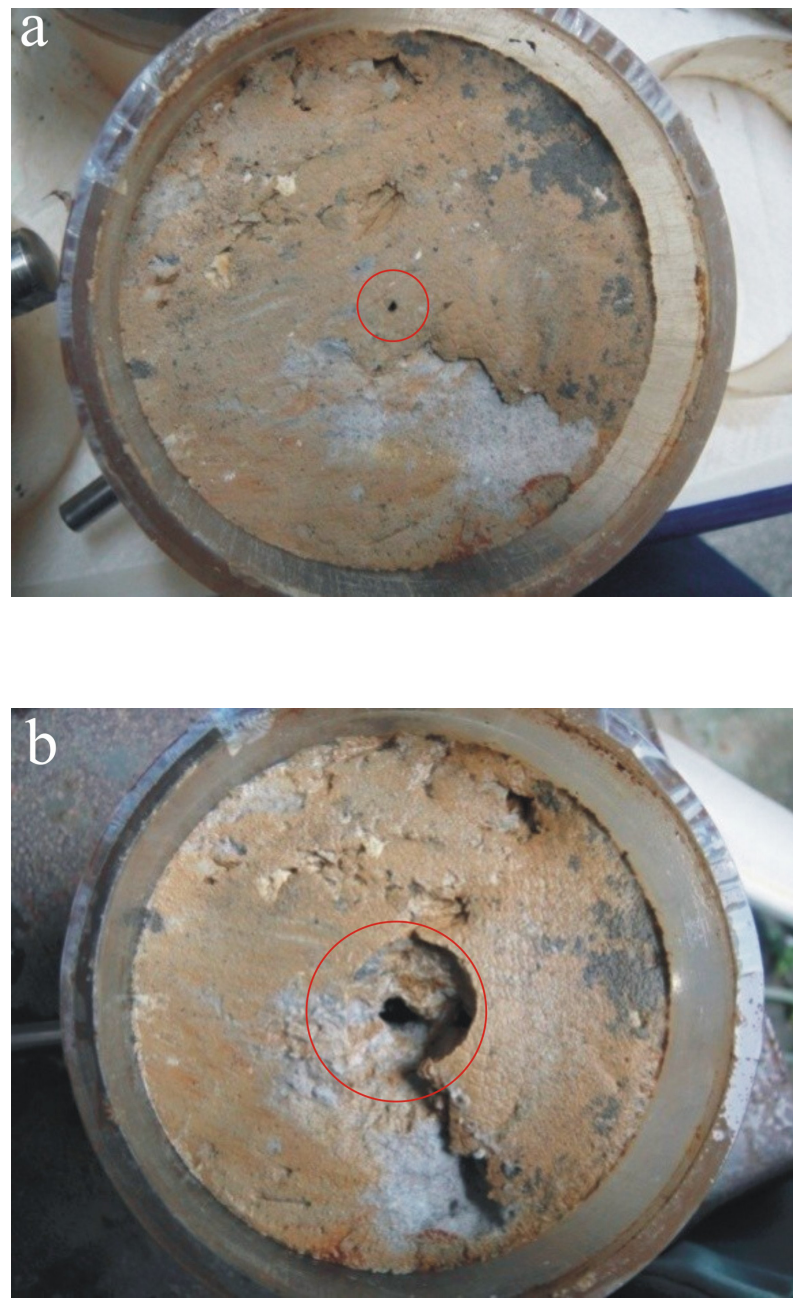

Figure 4. The change of the pipe diameter: $\boldsymbol{a}$. the black point in the red circle is the original pipe at diameter $1 \mathrm{~mm}$. b. showes the diameter changes at $3 \mathrm{~mm}$, which indicates the soil is broken.

$\begin{array}{ccc}\text { No. } & \begin{array}{c}\text { Critical seepage quantity } \\ (\mathrm{ml} / \mathrm{s})\end{array} & \begin{array}{c}\text { Water temperature } \\ \left({ }^{\circ} \mathrm{C}\right)\end{array} \\ \text { ZK5-1 } & 1.806 & 12.3 \\ \text { ZK5-2 } & 1.766 & 14.4 \\ \text { ZK5-3 } & 1.754 & 13.7\end{array}$

$\begin{array}{ccc}\begin{array}{c}\text { Dynamic viscosity coefficient } \\ \left(10^{-3} \mathrm{Kg} / \mathrm{ms}\right)\end{array} & \text { Reynolds } & \begin{array}{c}\text { No. } \\ (\mathrm{Pa})\end{array} \\ 1.225 & 1877 & 22.5 \\ 1.157 & 1943 & 20.8 \\ 1.179 & 1894 & 21.1\end{array}$

Table 1. The critical shear stress of samples. 
nification. Measured results presented the minimum at $15.75 \mathrm{~nm}$, the maximum at $72.08 \mathrm{~nm}$, and the average at $31.6 \mathrm{~nm}$. Therefore, $31.6 \mathrm{~nm}$ is the representative diameter of the tested sample.

\section{Critical velocity}

The critical velocity of suffosion is calculated by equation 11. Water temperature is monitored every $20 \mathrm{~min}-$ utes by an observation system in 2013 (all the time of the year). The max temperature is $22.6{ }^{\circ} \mathrm{C}$, and the min is $22.3{ }^{\circ} \mathrm{C}$. The average $22.38{ }^{\circ} \mathrm{C}$ is the water temperature in 2013 ; to obtain that $\mu$ is $0.948 \times 10-3 \mathrm{Kg} / \mathrm{ms}$. $\tau$ is $21.5 \mathrm{~Pa}$, and $\mathrm{d}$ is $31.6 \mathrm{~nm}$, therefore $\mathrm{Vcr}$ is $0.5375 \mathrm{~cm} / \mathrm{min}$ by equation 11 .

\section{Assessment}

The described test accesses quantitatively the risk of subsidence sinkholes via hydraulic condition. If groundwater velocity is more than (has risk) the critical velocity or not. Figure 5 shows the relationship between groundwater velocity and the critical velocity. It indicates that groundwater velocity is less than the critical velocity. As a result, Wuxuan has no risk of subsidence sinkholes caused by suffosion.

\section{Conclusion}

Suffosion is a main factor that leads to subsidence sinkholes. It derives the formula of the critical groundwater velocity of suffosion via the piping flow. Three samples

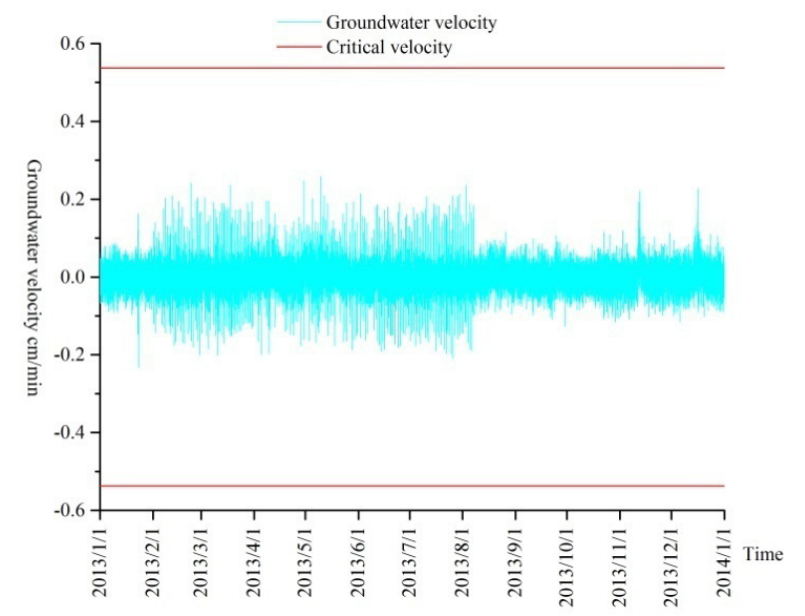

Figure 5. Velocity of groundwater in 2013 year. That the velocity is less than 0 indicates the moving direction of groundwater is upward. When the velocity is more than 0 , the moving direction is downward. from Wuxuan were tested by self-designed equipment to obtain the critical velocity, $0.5375 \mathrm{~cm} / \mathrm{min}$. Compared with the monitored hydrodynamic condition, groundwater velocity is less than the critical velocity. As a result, Wuxuan has no risk of subsidence sinkholes for suffosion.

\section{Reference}

Fuwei J, Mingtang L, Zhengde G, et al. 2014. Study on the critical hydraulic condition of forming soilcaves in karst area. Journal of engineering geology 22(sup): 77-82. 
